$$
t_{0} w_{2} \cdots=w_{2}^{m_{1}} t_{0} \cdots \text {. }
$$

By the definition of $q, w_{2}$ cannot be a left-factor of $t_{0}$. Therefore, by (3), $t_{0}$ must be a left-factor of $w_{2}$. We may write $w_{2}=t_{0} t_{1}$.

Equation (1) clearly has the form $v w_{1}=v^{\prime} w_{2}$, where $v, v^{\prime} \in F(\Sigma)$. This implies that $w_{2}$ is a right-factor of $w_{1}$. However, $w_{1}=w_{2}^{g-1} t_{0} t_{1} t_{0}$. Since the length of $t_{1} t_{0}$ is equal to the length of $w_{2}$, it follows that $w_{2}=t_{1} t_{0}$. Thus, $t_{1} t_{0}=t_{0} t_{1}$ and by the remark, $\rho\left(t_{0}\right)=\rho\left(t_{1}\right)=z$. This implies $\rho\left(w_{2}\right)=z$ and by (2) we also have $\rho\left(w_{1}\right)=z$. This completes the proof.

\title{
REFERENCES
}

1. P. M. Cohn, On subsemigroups of free semigroups, Proc. Amer. Math. Soc. 3 (1962), 347-351.

2. M. W. Curtis, On some commutative sets in a free monoid, Abstract 64T-203, Notices Amer. Math. Soc. 11 (1964), 250.

3. E. S. Lyapin, Semigroups, Translations of Mathematical Monographs, Vol. 3, Amer. Math. Soc., Providence, R. I., 1963.

4. R. C. Lyndon and M. P. Schutzenberger, The equation $a^{M}=b^{N} c^{P}$ in a free group. Michigan Math. J. 9 (1962), 289-298.

WESLEYAN UNIVERSITY

\section{CONDITIONAL INTEGRABILITY OVER MEASURE SPACES}

\author{
BY HUBERT HALKIN
}

\section{Communicated by P. R. Halmos, March 3, 1965}

Introduction. Let $E^{n}$ be the $n$-dimensional Euclidean space, $(A, a, \mu)$ be a measure space and $f$ be a measurable function from $A$ into $E^{n}$. According to the theory of integration the function $f$ is either integrable or not integrable. The aim of this note is to show that nonintegrable functions can be divided into two classes: the totally unintegrable functions and the conditionally integrable functions. Moreover we shall show that the conditionally integrable functions may be further characterized by an index of conditional integration which can take the values $1,2, \cdots, n$.

Notations. A subset $\mathscr{N}$ of $\mathcal{Q}$ is a nest if for any $N_{1}$ and $N_{2} \in \Re$ we have either $N_{1} \subset N_{2}$ or $N_{2} \subset N_{1}$. A nest $\mathscr{T}$ of $a$ is called a sweeping nest for the measure space $(A, Q, \mu)$ if for each set $D \in Q$ with $\mu(D)<\infty$ and for each $\epsilon>0$ there exists a set $N \in \Re$ such that 
$\mu(D \sim N) \leqq \epsilon$. If $f$ is a measurable function from $A$ into $E^{n}$ let $\mathscr{L}(f)$ be the subset of a such that for every $D \in \mathscr{L}(f)$ the function $f$ is integrable over $D$ and let $\Gamma(f)$ be the class of all sweeping nests of $(A, a, \mu)$ such that for any $\Re \in \Gamma$ we have $\Re \subset \mathscr{L}(f)$ and there exists a point $a \in E^{n}$ such that for all $\epsilon>0$ there exists a $D \in \mathscr{N}$ such that $\left|\int_{D * f} d \mu-a\right| \leqq \epsilon$ for all $D^{*} \in \mathfrak{N}$ with $D \subset D^{*}$. The point $a$ satisfying the preceding relation is uniquely defined and will be denoted $a(\mathfrak{T})$. Let $I(f)$ be the subset of $E^{n}$ defined by $I(f)=\{a(\mathfrak{x}): \Re \in \Gamma\}$. If the function $f$ is integrable over $(A, \mathfrak{Q}, \mu)$ the set $I(f)$ will have the single element $\int_{A} f d \mu$. The central result of this note states that:

THEOREM I. The set $I(f)$ is a linear variety.

By a linear variety we mean a set $I(f)$ such that if $a_{1}$ and $a_{2} \in I(f)$ then for every real $\lambda$ we have $a_{1}+\lambda\left(a_{2}-a_{1}\right) \in I(f)$.

The dimension of $I(f), d(I(f))$, can be used to characterize the integrability of the function $f$ with respect to the measure space $(A, \boldsymbol{Q}, \mu)$ :

If $d(I(f))=0$, i.e. the set $I(f)$ contains a single point, then the function $f$ is integrable over $(A, Q, \mu)$.

If $d(I(f))=-1$, i.e. the set $I(f)$ is empty, we say that the function $f$ is totally unintegrable over $(A, \propto, \mu)$.

If $1 \leqq d(I(f)) \leqq n$ we say that the function $f$ is conditionally integrable over $(A, a, \mu)$. In that case we shall call $d(I(f))$ the degree of conditional integrability of $f$ over $(A, \mathrm{Q}, \mu)$.

The proof of Theorem I is an easy application of Steinitz' Theorem, [1], on rearrangements of vector series. Conversely Steinitz' Theorem may be considered as a particular case of Theorem I.

\section{REFERENCE}

1. E. Steinitz, Bedingt konvergente Reihen und konvexe Systeme, J. Reine Angew. Math. 143 (1913), 128-175, ibid. 144 (1914), 1-40.

Bell Telephone laboratories, Incorporated 